FERMILAB-Conf-01/120-T

\title{
GUT implications from neutrino mass ${ }^{1}$
}

\author{
Carl H. Albright 2,3 \\ Fermi National Accelerator Laboratory, Batavia, IL 60510
}

\begin{abstract}
An overview is given of the experimental neutrino mixing results and types of neutrino models proposed, with special attention to the general features of various GUT models involving intra-family symmetries and horizontal flavor symmetries. Many of the features are then illustrated by a specific $S O(10)$ SUSY GUT model formulated by S.M. Barr and the author which can explain all four types of solar neutrino mixing solutions by various choices of the right-handed Majorana mass matrix. The quantitative nature of the model's large mixing angle solution is used to compare the reaches of a neutrino super beam and a neutrino factory for determining the small $U_{e 3}$ mixing matrix element.
\end{abstract}

Key words: GUT models; neutrino oscillations

\section{Introduction}

\subsection{Summary of neutrino mass and mixing data}

With $\Delta m_{i j}^{2} \equiv m_{i}^{2}-m_{j}^{2}$ and $U_{\ell i}$, one of the Maki-Nakagawa-Sakata (MNS) neutrino mixing matrix elements (11), the following information is known.

- Atmospheric Neutrinos

Interpretation of the data heavily favors $\nu_{\mu} \leftrightarrow \nu_{\tau}$ over $\nu_{\mu} \leftrightarrow \nu_{\text {sterile }}$ oscillations (2) with oscillation parameters

$$
\begin{aligned}
\Delta m_{32}^{2} & \simeq 3.2 \times 10^{-3} \mathrm{eV}^{2}, \\
\sin ^{2} 2 \theta_{\mathrm{atm}} & =4\left|U_{\mu 3}\right|^{2}\left|U_{\tau 3}\right|^{2}=1.000,(>0.89 @ 90 \% \text { c.l. })
\end{aligned}
$$

\section{- Solar Neutrinos}

Here the data favors $\nu_{e} \leftrightarrow \nu_{\mu}, \nu_{\tau}$ oscillations over $\nu_{e} \leftrightarrow \nu_{\text {sterile }}$ oscillations, but four solar mixing solutions were possible as reported by Gonzalez-Garcia

$\overline{1}$ Invited talk at the NuFACT'01 Workshop in Tsukuba, Japan, 24-30 May 2001.

2 electronic address: albright@fnal.gov

3 Supported in part by the U.S. Department of Energy 
at last year's Osaka HEP International Conference (3); i.e., the small mixing angle (SMA), the large mixing angle (LMA), and the (LOW) MSW (田) solutions, together with the quasi-vacuum (QVO) solution. In the (3 active, 0 sterile) neutrino framework, the best-fit mixing solutions obtained with $\sin ^{2} 2 \theta_{\text {sol }}=4\left|U_{e 1}\right|^{2}\left|U_{e 2}\right|^{2}$ were:

$$
\begin{aligned}
\text { SMA : } & \Delta m_{21}^{2} \simeq 5.0 \times 10^{-6} \mathrm{eV}^{2} \\
& \sin ^{2} 2 \theta_{\text {sol }} \simeq 0.0024, \tan ^{2} \theta \simeq 0.0006 \\
L M A: & \Delta m_{21}^{2} \simeq 3.2 \times 10^{-5} \mathrm{eV}^{2} \\
& \sin ^{2} 2 \theta_{\text {sol }} \simeq 0.75, \tan ^{2} \theta \simeq 0.33 \\
L O W: & \Delta m_{21}^{2} \simeq 1.0 \times 10^{-7} \mathrm{eV}^{2} \\
& \sin ^{2} 2 \theta_{\text {sol }} \simeq 0.96, \tan ^{2} \theta \simeq 0.67 \\
Q V O: & \Delta m_{21}^{2} \simeq 8.6 \times 10^{-10} \mathrm{eV}^{2} \\
& \sin ^{2} 2 \theta_{\text {sol }} \simeq 0.96, \tan ^{2} \theta \simeq 1.5
\end{aligned}
$$

A new analysis by the Super-Kamiokande collaboration based on 1258 days of data (5) now indicates that the LMA region is strongly preferred over the other regions with the best-fit point given by

$$
\begin{aligned}
L M A: & \Delta m_{21}^{2} \simeq 7 \times 10^{-5} \mathrm{eV}^{2} \\
& \sin ^{2} 2 \theta_{\text {sol }} \simeq 0.87, \tan ^{2} \theta \simeq 0.47 .
\end{aligned}
$$

With just three active neutrinos and maximal atmospheric mixing, to a good approximation one can simplify the general MNS mixing matrix to read

$$
U_{M N S} \simeq\left(\begin{array}{ccc}
c_{12} & s_{12} & 0 \\
-s_{12} / \sqrt{2} & c_{12} / \sqrt{2} & 1 / \sqrt{2} \\
s_{12} / \sqrt{2} & -c_{12} / \sqrt{2} & 1 / \sqrt{2}
\end{array}\right)
$$

where the mass eigenstates are given in terms of the flavor states by

$$
\begin{aligned}
& \nu_{3}=\frac{1}{\sqrt{2}}\left(\nu_{\mu}+\nu_{\tau}\right) \\
& \nu_{2}=\nu_{e} s_{12}+\frac{1}{\sqrt{2}}\left(\nu_{\mu}-\nu_{\tau}\right) c_{12} \\
& \nu_{1}=\nu_{e} c_{12}-\frac{1}{\sqrt{2}}\left(\nu_{\mu}-\nu_{\tau}\right) s_{12} .
\end{aligned}
$$

In the LMA case with the latest data given above, one then finds

$$
U_{M N S}^{(L M A)} \simeq\left(\begin{array}{ccc}
0.825 & 0.565 & 0 \\
-0.400 & 0.583 & 0.707 \\
0.583 & -0.400 & 0.707
\end{array}\right)
$$


Note that with maximal atmospheric mixing, unitarity forces $\theta_{13}=0^{\circ}$; in fact, a primary physics goal of a neutrino factory is to determine $U_{e 3}$ and its departure from zero. While solar neutrino mixing is close to maximal, strictly maximal mixing is presently excluded at the $95 \%$ c.l. If this persists, it may result in a severe test for models to predict the deviation from maximal mixing.

\subsection{Types of neutrino models}

The general neutrino mass matrix in a basis with $n_{L}$ left-handed fields and $n_{R}=n_{L}^{c}$ right-handed or left-handed conjugate fields has the complex symmetric form

$$
\mathbf{M}_{\nu}=\left(\begin{array}{cc}
M_{L} & N^{T} \\
N & M_{R}
\end{array}\right)
$$

where $M_{L}$ is the left-handed Majorana mass matrix, $N$ and $N^{T}$ the Dirac mass matrix and its transpose, and $M_{R}$ is the right-handed Majorana mass matrix. Models which appear in the literaturet can generally be placed into three classes as follows:

- Models with only left-handed neutrinos present

Models of this type are variations of the Zee model (7), where ultralight neutrinos arise from non-renormalizable contributions involving some undetermined high mass scale. Lepton number is violated by two units, or an $L=$ -2 isovector Higgs field is introduced. The combination $L^{\prime} \equiv L_{e}-L_{\mu}-L_{\tau}$ is often taken to be conserved.

- Models with both left- and right-handed neutrinos present With $M_{L}=0$, the seesaw mechanism yields ultralight neutrino masses provided the right-handed masses are in the range of $10^{5}-10^{15} \mathrm{GeV}$. Such masses are naturally obtained in GUT models with $\Lambda_{G U T}=2 \times 10^{16} \mathrm{GeV}$.

\section{- Models with neutrinos in higher dimensions}

Right-handed neutrinos which are singlets under all gauge symmetries can enter the bulk with many Kalusa-Klein states present. With large extra dimensions and the compactification scale much lower than the string scale, a modified seesaw mechanism can generate ultralight neutrino masses (8).

\section{Features of various GUT models}

Restricting our attention to the second class of models, we note the intrafamily symmetry specified by a GUT model provides a unified treatment of quarks and leptons as (some) quarks and leptons are placed in the same multiplets. For example, the representation content of three familiar GUTs is listed below:

$\overline{4}$ More complete surveys can be found in (6). 


$$
\begin{aligned}
S U(5): & \left(u_{\alpha}, d_{\alpha}, u_{\alpha}^{c}, \ell^{c}\right)_{i} \subset \mathbf{1 0}_{i} \\
& \left(d_{\alpha}^{c}, \ell, \nu_{\ell}\right)_{i} \subset \overline{\mathbf{5}}_{i}, \quad \alpha=r, b, g ; i=1,2,3 \\
& \left(\nu_{\ell}^{c}\right)_{i} \subset \mathbf{1}_{i} \\
S O(10): & \left(u_{\alpha}, d_{\alpha}, u_{\alpha}^{c}, d_{\alpha}^{c}, \ell, \ell^{c}, \nu_{\ell}, \nu_{\ell}^{c}\right)_{i} \subset \mathbf{1 6}_{i} \\
E_{6}: & \left(u_{\alpha}, d_{\alpha}, u_{\alpha}^{c}, d_{\alpha}^{c}, \ell, \ell^{c}, \nu_{\ell}, \nu_{\ell}^{c}\right)_{i}, \\
& \left(D_{\alpha}, D_{\alpha}^{c}, E, E^{c}, N, N^{c}\right)_{i}, n_{i} \subset \mathbf{2 7}_{i}
\end{aligned}
$$

Sterile neutrinos can appear non-trivially in $E_{6}$ or as isolated singlets in $S U(5)$ or $S O(10)$.

On the other hand, a specified horizontal flavor symmetry enables one to connect comparable flavors in different families which allows a mass hierarchy to exist among the families. The flavor symmetry may be discrete, such as $Z_{2}, S_{3}, Z_{2} \times Z_{2}$ etc., and results in multiplicative quantum numbers. A continuous flavor symmetry, such as $U(1), U(2), S U(3)$ etc., results in additive quantum numbers and may be global or local (and possibly anomalous).

With a GUT family symmetry, some or all of the flavor bases are related for the up and down quark, charged lepton and neutrino Dirac mass matrices, hereafter denoted by $U, D, L$, and $N$. As such, the Yukawa interactions in GUT models are typically not diagonal in flavor space. Contrast this with models with no grand unification, where the quark and lepton sectors can be treated independently and some matrices can be arbitrarily assumed diagonal.

In unbroken $S U(5), L=D^{T}$, but $N$ and $D$ are unrelated, while the righthanded Majorana mass matrix, $M_{R}$ may or may not exist. This tends to provide a lot of freedom for the model builder as different flavor charges can be assigned to each $\mathbf{1 0}, \overline{\mathbf{5}}$ and $\mathbf{1}$ in the same family.

In unbroken $S O(10), U=D=L=N$ as all left-handed quarks and leptons belonging to the same family have the same flavor charge, while $M_{R}$ exists and is independent of the others due to its different Higgs VEV structure. With $S U(5)$ and $S O(10)$ broken at the GUT scale, and the Higgs fields as well as the fermion fields carrying horizontal flavor quantum numbers, a rather complex set of mass matrix textures can emerge.

In $E_{6}$ eleven extra states are present in each fundamental 27 which must be made heavy, aside possibly from 1 or 2 light sterile neutrinos per family. 


\section{Symmetry breaking in $S O(10)$}

Now restricting our attention to $S O(10)$, we note that to break $S O(10)$ to the $\mathrm{SM}$, the rank must be reduced from 5 to 4 typically along one of the chains:

$$
\begin{aligned}
S O(10) & \rightarrow S U(5) \times U(1) \rightarrow S U(5) \rightarrow \mathrm{SM}, \\
S O(10) & \rightarrow S U(4) \times S U(2)_{L} \times S U(2)_{R} \\
& \rightarrow S U(3) \times S U(2)_{L} \times S U(2)_{R} \rightarrow \mathrm{SM}
\end{aligned}
$$

Among the possible $S O(10)$ Higgs VEVs in the first breaking chain at the GUT scale are:

$\left\langle\mathbf{4 5}_{\mathbf{H}}\right\rangle$ which can point in the $I_{3 R}, Y$ or $B-L$ direction, but these do not reduce the rank;

$\left\langle 1\left(\mathbf{1 6}_{\mathbf{H}}\right)\right\rangle$ which breaks $S O(10) \rightarrow S U(5)$ by reducing the rank;

$\left\langle\mathbf{4 5}_{\mathbf{H}}\right\rangle_{B-L}+\left\langle 1\left(\mathbf{1 6}_{\mathbf{H}}\right)\right\rangle$ which breaks $S O(10) \rightarrow$ SM.

As for the electroweak Higgs doublets, they can appear in the 5 and $\overline{5}$ representations of $S U(5)$ and will break $\mathrm{SM} \rightarrow S U(3)_{c} \times U(1)_{\text {em }}$ at the electroweak scale, provided they remain light while the Higgs color triplets get massive at $\Lambda_{G U T}$. This is known as the doublet-triplet splitting problem. In the standard procedure, the 5 and $\overline{5}$ are placed in the same $\mathbf{1 0}_{\mathbf{H}}$ of $S O(10)$ enabling Yukawa coupling unification with $\tan \beta \simeq 55$. However, another possibility is to place the 5 in the $\mathbf{1 0}_{\mathbf{H}}$ while the $\overline{5}$ belongs to a linear combination of the $\mathbf{1 0}_{\mathbf{H}}$ and a $\mathbf{1 6}_{\mathbf{H}}$. This enables Yukawa unification with $1 \lesssim \tan \beta \lesssim 55$. In any case, only two Higgs doublets can survive down to the electroweak scale for proper gauge coupling unification with $\sin ^{2} \theta_{W} \sim 0.2315$.

GUT models then differ by their choice of unification group, symmetry-breaking schemes and assigned flavor symmetries. Among $S O(10)$ GUT models, the following flavor symmetries appear in the literature:

$$
\begin{array}{ll}
U(1) & \text { Babu, Pati, Wilczek (9) } \\
U(1) \times Z_{2} \times Z_{2} & \text { Albright, Babu, Barr (10) } \\
S U(2) \times Z_{2} \times Z_{2} \times Z_{2} & \text { Chen, Mahanthappa (11) } \\
U(2) \times U(1)^{n} & \text { Blazek, Raby, Toby (12) } \\
S U(3) & \text { Berezhiani, Rossi (13) }
\end{array}
$$

All rely on the seesaw mechanism (14), $M_{\nu}=-N^{T} M_{R} N$, to obtain the light effective LH Majorana mass matrix, but $M_{R}$ may be generated with a $\left\langle 1\left(\overline{\mathbf{1 2 6}}_{\mathbf{H}}\right)\right\rangle$ or a pair of $\left\langle 1\left(\overline{\mathbf{1 6}}_{\mathbf{H}}\right)\right\rangle$ 's. With $U_{M N S}=U_{L}^{\dagger} U_{\nu}$, all models generate the maximal atmospheric $\nu_{\mu}-\nu_{\tau}$ mixing either by a special feature of $N$, a special feature of 
$L$, or the combined effect of $N$ and $M_{R}$ in the seesaw mechanism. Most models easily accommodate the SMA solar solution, while some can accommodate the QVO or LOW solution as well. However, most have great difficulty with, or find it impossible to explain, the LMA solution, since fine tuning is required. This is especially true of models which require special features of $N$ and/or $M_{R}$ to get maximal atmospheric mixing.

\section{$4 S O(10)$ SUSY GUT model with $U(1) \times Z_{2} \times Z_{2}$ flavor symmetry}

We now illustrate a model, developed in collaboration with S.M. Barr (15), which is particularly useful in that it is quantitatively predictive, can explain the LMA solution, and can be used to assess the need for a neutrino factory.

It is based on a minimum set of Higgs fields which solves the doublet-triplet splitting problem with just one $45_{H}$ whose VEV points in the $B-L$ direction and has no higher rank representations. Two pairs of $\mathbf{1 6}_{H}, \overline{\mathbf{1 6}}_{H}$ 's stabilize the solution (16). Several Higgs in the $\mathbf{1 0}_{H}$ representations together with Higgs singlets are also present. The combination of VEVs, $\left\langle\mathbf{4} \mathbf{5}_{\mathbf{H}}\right\rangle_{B-L},\left\langle 1\left(\mathbf{1 6}_{\mathbf{H}}\right)\right\rangle$ and $\left\langle 1\left(\overline{\mathbf{1 6}}_{\mathbf{H}}\right)\right\rangle$ break $S O(10)$ to the SM. The electroweak VEVs arise from $v_{u}=$ $\left\langle 5\left(\mathbf{1 0}_{\mathbf{H}}\right)\right\rangle$ and $v_{d}=\left\langle\overline{5}\left(\mathbf{1 0}_{\mathbf{H}}\right)\right\rangle \cos \gamma+\left\langle\overline{5}\left(\mathbf{1} \mathbf{6}_{\mathbf{H}}^{\prime}\right)\right\rangle \sin \gamma$, while the combination orthogonal to $v_{d}$ gets massive at the GUT scale. The Higgs superpotential in this model exhibits the $U(1) \times Z_{2} \times Z_{2}$ flavor symmetry.

In addition, matter superfields appear in the following representations:

$16_{1}, 16_{2}, 16_{3} ; 16, \overline{16}, 16^{\prime}, \overline{16^{\prime}}, 10_{1}, 10_{2}$, and 1 's, where all but the $16_{\mathrm{i}}, i=$ $1,2,3$ get superheavy and are integrated out.

The Dirac mass matrices are found to be

$$
\begin{aligned}
& U=\left(\begin{array}{ccc}
\eta & 0 & 0 \\
0 & 0 & \epsilon / 3 \\
0 & -\epsilon / 3 & 1
\end{array}\right) M_{U}, \quad D=\left(\begin{array}{ccc}
0 & \delta & \delta^{\prime} e^{i \phi} \\
\delta & 0 & \sigma+\epsilon / 3 \\
\delta^{\prime} e^{i \phi} & -\epsilon / 3 & 1
\end{array}\right) M_{D}, \\
& N=\left(\begin{array}{ccc}
\eta & 0 & 0 \\
0 & 0 & -\epsilon \\
0 & \epsilon & 1
\end{array}\right) M_{U}, \quad L=\left(\begin{array}{ccc}
0 & \delta & \delta^{\prime} e^{i \phi} \\
\delta & 0 & -\epsilon \\
\delta^{\prime} e^{i \phi} & \sigma+\epsilon & 1
\end{array}\right) M_{D},
\end{aligned}
$$

where

$$
\begin{aligned}
M_{U} & \simeq 113 \mathrm{GeV}, & M_{D} & \simeq 1 \mathrm{GeV}, \\
\sigma & =1.78, & \epsilon & =0.145, \\
\delta & =0.0086, & \delta^{\prime} & =0.0079, \\
\phi & =54^{\circ}, & \eta & =8 \times 10^{-6}
\end{aligned}
$$

are input parameters defined at the GUT scale to fit the low scale observables after evolution downward from $\Lambda_{G U T}$. 
The above textures were obtained by imposing the Georgi-Jarlskog relations (17) at $\Lambda_{G U T}, m_{s}^{0} \simeq m_{\mu}^{0} / 3, m_{d}^{0} \simeq 3 m_{e}^{0}$ with Yukawa coupling unification holding for $\tan \beta \sim 5$. The matrix element contributions can be neatly understood in terms of Froggatt-Nielsen diagrams (18). In particular, " 1 ' $s$ " are obtained from the $\mathbf{1 6}_{\mathbf{3}} \cdot \mathbf{1 6}_{\mathbf{3}} \cdot \mathbf{1 0}_{\mathbf{H}}$ vertices; the " $\epsilon$ " terms are obtained from diagrams exhibiting the $\left\langle\mathbf{4 5}_{\mathbf{H}}\right\rangle_{B-L}$ suppression; while the " $\sigma$ " terms arise from the $\mathbf{1 6}_{\mathbf{2}} \cdot \mathbf{1 6}_{\mathbf{H}} \cdot \mathbf{1 6}_{\mathbf{H}}^{\prime} \cdot \mathbf{1 6}_{\mathbf{3}}$ effective operator which contributes only to $D$ and $L$ in the lop-sided fashion indicated. The other entries arise from more complex diagrams (15).

All nine quark and charged lepton masses plus the three CKM angles and CP phase are well-fitted with the eight input parameters. The vertex of the CKM unitary triangle occurs at the center of the presently allowed region with $\sin 2 \beta \simeq 0.65$. The Hermitian matrices $U^{\dagger} U, D^{\dagger} D$, and $N^{\dagger} N$ are diagonalized with small left-handed rotations, while $L^{\dagger} L$ is diagonalized by a large lefthanded rotation. This neatly accounts for the fact that $V_{c b}=\left(U_{U}^{\dagger} U_{D}\right)_{c b}$ is small, while $U_{\mu 3}=\left(U_{L}^{\dagger} U_{\nu}\right)_{\mu 3}$ is large for any reasonable $M_{R}$.

Since the solar and atmospheric mixings are essentially decoupled in the model, the structure of the right-handed Majorana mass matrix determines the type of $\nu_{e} \leftrightarrow \nu_{\mu}, \nu_{\tau}$ solar neutrino mixing.

- The SMA Solar Neutrino Solution can be obtained with

$$
M_{R}=\left(\begin{array}{ccc}
C & 0 & 0 \\
0 & B \epsilon^{2} & 0 \\
0 & 0 & 1
\end{array}\right) \Lambda_{R}
$$

and $B=-1.9, C=5 \times 10^{-8}, \Lambda_{R}=1.2 \times 10^{14} \mathrm{GeV}$; for small mixing in the 1-2 sector of the matrices arises since $N$ and $L$ are nearly diagonal there.

- The QVO Solar Neutrino Solution can be obtained with

$$
M_{R}=\left(\begin{array}{ccc}
0 & A \epsilon^{3} & 0 \\
A \epsilon^{3} & 0 & 0 \\
0 & 0 & 1
\end{array}\right) \Lambda_{R}
$$

and $A=0.05, \Lambda_{R}=2.4 \times 10^{14} \mathrm{GeV}$, which leads to a pair of pseudo-Dirac neutrinos.

- The LMA Solar Neutrino Solution, unlike the others, requires finetuning for $M_{R}$ and a nearly hierarchical texture:

$$
M_{R}=\left(\begin{array}{ccc}
c^{2} \eta^{2} & -b \epsilon \eta & a \eta \\
-b \epsilon \eta & \epsilon^{2} & -\epsilon \\
a \eta & -\epsilon & 1
\end{array}\right) \Lambda_{R}
$$

in terms of parameters $\epsilon$ and $\eta$ introduced in the Dirac sector. Note that the 2-3 subsector has zero determinant and is closely related to that of $N$, as can also be understood in terms of Froggatt-Nielsen diagrams. 
With $a=1, b=c=2$ and $\Lambda_{R}=2.5 \times 10^{14} \mathrm{GeV}$, by the seesaw mechanism the light neutrino mass matrix becomes

$$
M_{\nu}=\left(\begin{array}{ccc}
0 & -\epsilon & 0 \\
-\epsilon & 0 & 2 \epsilon \\
0 & 2 \epsilon & 1
\end{array}\right) M_{U}^{2} / \Lambda_{R}
$$

with three texture zeros, which leads to

$$
\begin{array}{ll}
M_{1}=M_{2}=2.8 \times 10^{8} \mathrm{GeV}, & M_{3}=2.5 \times 10^{14} \mathrm{GeV}, \\
\Delta m_{32}^{2}=3.2 \times 10^{-3} \mathrm{eV}^{2}, & \sin ^{2} 2 \theta_{\mathrm{atm}}=0.994 \\
\Delta m_{21}^{2}=6.5 \times 10^{-5} \mathrm{eV}^{2}, & \sin ^{2} 2 \theta_{\text {sol }}=0.88 \\
U_{e 3}=-0.014, & \sin ^{2} 2 \theta_{\text {reac }}=0.0008
\end{array}
$$

which compares favorably with the present S-K best-fit point in the LMA region cited in the introduction. Note also that $\sin ^{2} 2 \theta_{\text {atm }}$ is extremely close to maximal. In fact, the whole presently-allowed LMA region can be covered with $1.0 \lesssim a \lesssim 2.5,1.8 \lesssim b=c \lesssim 5.2$ as shown in Fig. 1 , where contours of constant $\sin ^{2} 2 \theta_{12}$ and $\sin ^{2} 2 \theta_{13}$ are plotted. From this Figure, the advantage of a neutrino factory over a superbeam facility is apparent for this model. Other plots of similar interest have been obtained by the author in collaboration with S. Geer (19).

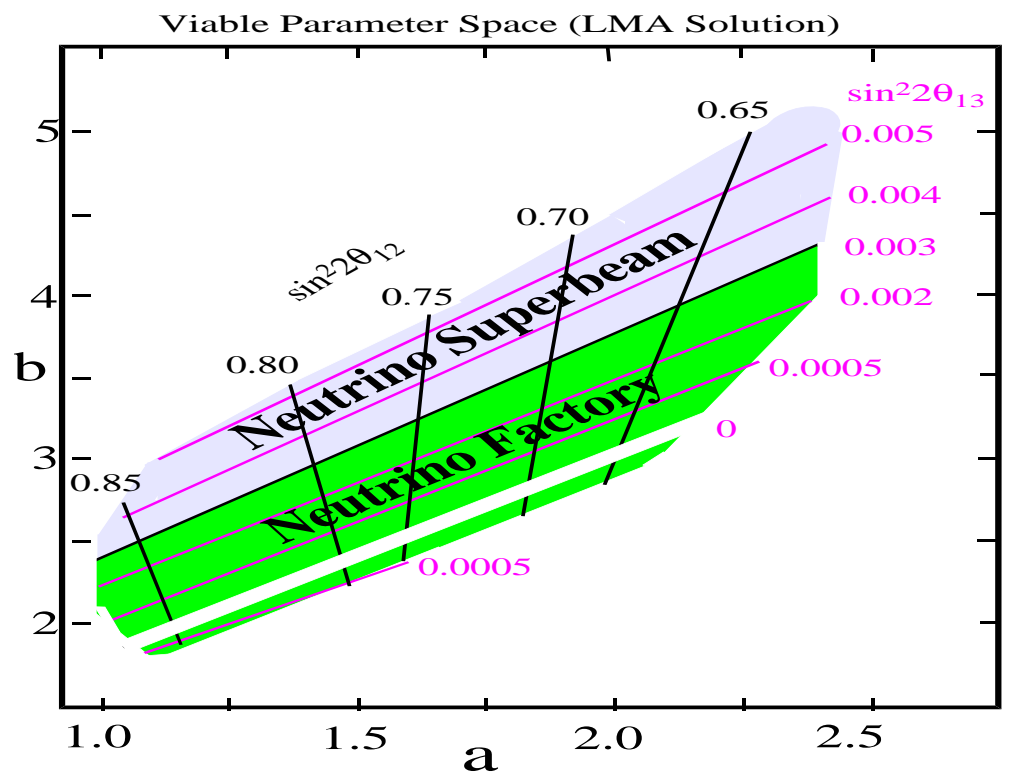

Fig. 1. Input parameter space with $b=c$ vs. $a$ in the right-handed Majorana mass matrix for the LMA solution. Contours of constant $\sin ^{2} 2 \theta_{12}$ and $\sin ^{2} 2 \theta_{13}$ are indicated with the reaches of a neutrino superbeam and that for a neutrino factory highlighted. Only the thin sliver around $\sin ^{2} 2 \theta_{13}=0$ corresponding to maximal atmospheric neutrino mixing is inaccessible to a neutrino factory. 


\section{Summary}

We have seen that $S O(10)$ SUSY GUT models can explain the observed atmospheric and solar neutrino oscillation data within the (3 active, 0 sterile) neutrino framework. Unfortunately, there is no strong preference for any particular solar neutrino solution, though the SMA, QVO and LOW solutions are easiest to obtain, with the LMA solution requiring fine-tuning. In the model described, that fine-tuning can be understood in terms of Froggatt-Nielsen diagrams. Finally we noted that a neutrino factory is essentially required, in order to determine $U_{e 3}$ for the present fully-allowed LMA region.

\section{References}

[1] Z. Maki, M. Nakagawa, and S. Sakata, Prog. Theor. Phys. 28 (1962) 870.

[2] S. Fukuda et al., Super-Kamiokande Collab., Phys. Rev. Lett. 85 (2000) 3999.

[3] M.C. Gonzalez-Garcia, Nucl. Phys. Proc. Suppl. 95 (2001) 100.

[4] L. Wolfenstein, Phys. Rev. D 17 (1978) 2369; S.P. Mikheyev and A. Yu. Smirnov, Yad. Fiz. 42 (1985) 1441, [Sov. J. Nucl. Phys. 42 (1985) 913].

[5] S. Fukuda et al., Super-Kamiokande Collab., hep-ph/0103033.

[6] S.M. Barr and I. Dorsner, Nucl. Phys. B 585 (2000) 79; G. Altarelli, hep-ph/0106085.

[7] A. Zee, Phys. Lett. B 93 (1980) 389.

[8] K.R. Dienes, E. Dudas, and T. Gherghetta, Nucl. Phys. B 557 (1999) 25.

[9] K.S. Babu, J.C. Pati, and F. Wilczek, Nucl. Phys. B 566 (2000) 33.

[10] C.H. Albright, K.S. Babu, and S.M. Barr, Phys. Rev. Lett. 81 (1998) 1167.

[11] M.-C. Chen and K.T. Mahanthappa, Phys. Rev. D 62 (2000) 113007.

[12] T. Blazek, S. Raby, and K. Tobe, Phys. Rev. D 62 (2000) 055001.

[13] Z. Berezhiani and A. Rossi, Nucl. Phys. B 594 (2001) 113.

[14] M. Gell-Mann, P. Ramond, and R. Slansky, Report No. CALT-68-709: Supergravity (North Holland, Amsterdam, 1979); T. Yanagida, in Proceedings of the Workshop on Unified Theory and Baryon Number of the Universe, KEK, Japan, 1979, unpublished.

[15] C.H. Albright and S.M. Barr, Phys. Rev. Lett. 85 (2000) 244; Phys. Rev. D 62 (2000) 093008; hep-ph/0104294.

[16] S.M. Barr and S. Raby, Phys. Rev. Lett. 79 (1997) 4748.

[17] H. Georgi and C. Jarlskog, Phys. Lett. B 86 (1979) 297.

[18] C.D. Froggatt and H.B. Nielsen, Nucl. Phys. B 147 (1979) 277.

[19] C.H. Albright and S. Geer, this workshop and in preparation. 